\title{
Ramsey-Type Results for Geometric Graphs, II*
}

\author{
Gy. Károlyi, ${ }^{1,2}$ J. Pach, ${ }^{3,4}$ G. Tóth,,${ }^{4,5}$ and P. Valtr ${ }^{6}$ \\ ${ }^{1}$ Eötvös Loránd University, \\ Múzeum krt. 6-8, 1088 Budapest, Hungary \\ karolyi@cs.elte.hu \\ ${ }^{2}$ ETH-Zentrum, \\ CH-8092 Zürich, Switzerland \\ ${ }^{3}$ City College, C.U.N.Y., \\ New York, NY 10031, USA \\ ${ }^{4}$ Courant Institute, New York University, \\ 251 Mercer Street, New York, NY 10012, USA \\ \{pach, toth\}@cims.nyu.edu \\ ${ }^{5}$ Mathematical Institute, Hungarian Academy of Sciences, \\ P.O.B. 127, H-1364 Budapest, Hungary \\ geza@math-inst.hu \\ ${ }^{6}$ Department of Applied Mathematics, Charles University, \\ Malostranské nám. 25, 11800 Praha 1, Czech Republic \\ valtr@kam.ms.mff.cuni.cz
}

\begin{abstract}
We show that for any two-coloring of the $\left(\begin{array}{l}n \\ 2\end{array}\right)$ segments determined by $n$ points in the plane, one of the color classes contains noncrossing cycles of lengths $3,4, \ldots,\lfloor\sqrt{n / 2}\rfloor$. This result is tight up to a multiplicative constant. Under the same assumptions, we also prove that there is a noncrossing path of length $\Omega\left(n^{2 / 3}\right)$, all of whose edges are of the same color. In the special case when the $n$ points are in convex position, we find longer monochromatic noncrossing paths, of length $\lfloor(n+1) / 2\rfloor$. This bound cannot be improved. We also discuss some related problems and generalizations. In particular, we give sharp estimates for the largest number of disjoint monochromatic triangles that can always be selected from our segments.
\end{abstract}

* Gy. Károlyi was supported by NSF Grant DMS-9304580, the Alfred Sloan Foundation, and DIMACS under NSF Grant STC-91-19999. J. Pach was supported by NSF Grant CCR-94-24398, PSC-CUNY Research Award 663472, and OTKA-T-020914. G. Tóth was supported by OTKA-14220, OTKA-T-020914, and the Alfred Sloan Foundation. P. Valtr was supported by the Czech Republic Grant GAČR 0194, Charles University Grants GAUK 193/1996 and 194/1996, and by DIMACS Center. 


\section{Introduction}

A geometric graph is a graph drawn in the plane so that every vertex corresponds to a point, and every edge is a closed straight-line segment connecting two vertices but not passing through a third. The $\left(\begin{array}{l}n \\ 2\end{array}\right)$ segments determined by $n$ points in the plane, no three of which are collinear, form a complete geometric graph with $n$ vertices (see [PA]). In classical Ramsey theory, we want to find large monochromatic subgraphs in a complete graph whose edges are colored with several colors [B], [GRS]. Most questions of this type can be formulated for complete geometric graphs, where the monochromatic subgraphs are required to satisfy certain geometric conditions. The investigation of these problems was initiated in [KPT].

A subgraph of a geometric graph is said to be noncrossing, if no two of its edges have an interior point in common. In this paper we show how to find large noncrossing monochromatic paths and cycles in a geometric graph whose edges are colored with two colors.

Theorem 1.1. For any two-coloring of the edges of a complete geometric graph with $n$ vertices, there exist monochromatic noncrossing cycles of length $3,4, \ldots,\lfloor\sqrt{n / 2}\rfloor$, having the same color. The order of magnitude of this bound cannot be improved.

Theorem 1.2. For any two-coloring of the edges of a complete geometric graph with $n$ vertices, there exists a noncrossing path of length $\Omega\left(n^{2 / 3}\right)$, all of whose edges are of the same color.

The last result improves the bound $\Omega\left(n^{1 / 2}\right)$ established in [KPT]. It is very likely that the $\Omega\left(n^{2 / 3}\right)$ bound in Theorem 1.2 can be further improved to (nearly) linear. We can verify this conjecture for convex geometric graphs, i.e., for geometric graphs whose $n$ points form the vertex set of a convex polygon.

Theorem 1.3. For any two-coloring of the edges of a complete convex geometric graph with $n \geq 3$ vertices, there exists a noncrossing monochromatic path of length $\lfloor(n+1) / 2\rfloor$. This bound cannot be improved.

The proof of Theorem 1.3 uses the following unpublished result of Micha Perles, whose proof is included.

Theorem 1.4 (Perles). If a convex geometric graph of $n \geq k+1$ vertices has more than $\lfloor(k-1) n / 2\rfloor$ edges, then it contains a noncrossing path of length $k$. This bound cannot be improved.

A graph is called a caterpillar if it is a tree containing no three edge disjoint paths of length 2 starting at the same vertex. In other words, a caterpillar is a path with some edges attached to it. In fact, Perles proved that under the conditions of Theorem 1.4, a 
noncrossing subgraph isomorphic to any given caterpillar of $k+1$ vertices can always be found.

The proofs of Theorems 1.1-1.4 can be turned into $O\left(n^{2}\right)$-time algorithms to find monochromatic cycles (resp. paths) with the required properties.

The above results can be rephrased using the following notation. Let $\mathcal{G}$ be a class of (so-called forbidden) geometric subgraphs. We want to determine the smallest number $R=R(\mathcal{G})$ with the property that every complete geometric graph with $R$ vertices, whose edges are colored with two colors, contains a monochromatic subgraph belonging to $\mathcal{G}$. If we restrict our attention to convex geometric graphs, then the corresponding function is denoted by $R_{\mathrm{c}}(\mathcal{G})$. Clearly, $R_{\mathrm{c}}(\mathcal{G}) \leq R(\mathcal{G})$.

For any positive integer $k$, let $k \mathcal{G}$ denote the class of all geometric graphs that can be obtained by taking the union of $k$ pairwise disjoint members of $\mathcal{G}$.

Theorem 1.5. Let $\mathcal{G}$ be any class of geometric graphs, each of which has at least two vertices.

(i) If $k$ is a power of 2 , then

$$
R(k \mathcal{G}) \leq(R(\mathcal{G})+1) k-1
$$

(ii) For any $k>0$,

$$
R(k \mathcal{G}) \leq\left\lceil\frac{3(R(\mathcal{G})+1)}{2}\right\rceil k-\left\lceil\frac{R(\mathcal{G})+1}{2}\right\rceil .
$$

(iii) For any $k>0$,

$$
R_{\mathrm{c}}(k \mathcal{G}) \leq\left(R_{\mathrm{c}}(\mathcal{G})+1\right) k-1
$$

In particular, if $\mathcal{G}=\mathcal{T}$ is the class of triangles, we have $R(\mathcal{T})=6$. Thus, by Theorem 1.5(i), we obtain

$$
R(k \mathcal{T}) \leq 7 k-1,
$$

provided that $k$ is a power of 2 . The following theorem shows that this result cannot be improved.

Theorem 1.6. Let $\mathcal{T}$ denote the class of triangles and let $k$ be a positive integer. Then

$$
R_{\Delta}(k):=R(k \mathcal{T}) \geq(R(\mathcal{T})+1) k-1=7 k-1 .
$$

Using the approach of [KPTT], an $O\left(n^{\log \log n+2}\right)$-time algorithm can be designed to find $k$ pairwise noncrossing triangles of the same color in any complete geometric graph of $n=7 k-1$ vertices, whose edges are colored with two colors, provided that $k$ is a power of 2. (Note that in an abstract graph, $\lfloor n / 5\rfloor$ pairwise vertex-disjoint triangles of the same color can always be found [BES].)

The paper is organized as follows. Sections 2 and 3 contain the proofs of Theorems 1.1 and 1.2, respectively. Theorems 1.3 and 1.4 are proved in Section 4, while Sections 5 and 6 contain the proofs of Theorems 1.5 and 1.6, respectively. 


\section{Cycles-Proof of Theorem 1.1}

Theorem 1.1 is an immediate consequence of the following result.

Theorem 2.1. Let $\mathcal{C}_{k}$ be the class of all noncrossing cycles of length $k$, and let $\mathcal{D}_{k}$ be the class of all noncrossing cycles of length $k$, triangulated from a vertex. Then

$$
\Omega\left(k^{2}\right)=R_{\mathrm{c}}\left(\mathcal{C}_{k}\right) \leq R\left(\mathcal{C}_{k}\right) \leq R\left(\mathcal{D}_{k}\right)=O\left(k^{2}\right) .
$$

Proof. First we prove that $R_{\mathrm{c}}\left(\mathcal{C}_{k}\right)>(k-1)^{2}$.

Take $(k-1)^{2}$ points on a circle and partition them into $k-1$ groups, each containing $k-1$ consecutive points. Color with red all edges between points in different groups, and color with blue all edges between points belonging to the same group.

Any red noncrossing cycle contains at most one point from each group, hence it cannot have more than $k-1$ points. On the other hand, all vertices of a blue cycle are from the same group, so there is no blue cycle with more than $k-1$ points.

Next we show that $R\left(\mathcal{D}_{k}\right) \leq 2(k-1)(k-2)+2$.

Let $P$ denote the vertex set of a complete geometric graph $G$ of $2(k-1)(k-2)+2$ vertices, whose edges are colored with red and blue. Let $p$ be a vertex of the convex hull of $P$. Since there are $2(k-1)(k-2)+1$ edges incident to $p$, at least $(k-1)(k-2)+1$ of them are of the same color, say, red. Let $p_{1}, p_{2}, \ldots, p_{(k-1)(k-2)+1}$ be vertices of $G$, listed in clockwise order of visibility from $p$, such that each edge $p p_{i}$ is red. A path $p_{i_{1}} p_{i_{2}} \cdots p_{i_{j}}$ is said to be monotone if $i_{1}<i_{2}<\cdots<i_{j}$.

Define a partial ordering of the vertices $p_{1}, p_{2}, \ldots, p_{(k-1)(k-2)+1}$, as follows. Let $p_{i}<p_{j}$ if $i<j$ and there is a monotone red path connecting $p_{i}$ to $p_{j}$. By Dilworth's theorem [D], there are either $k-1$ elements that form a totally ordered subset, or $k$ elements that are pairwise incomparable. In the first case, there is a monotone red path $q_{1} q_{2} \cdots q_{k-1}$ and we can complete it to a noncrossing red cycle $p q_{1} q_{2} \cdots q_{k-1}$ of size $k$, together with the corresponding diagonals from $p$. In the second case, there is a complete blue subgraph of $k$ vertices $q_{1}, q_{2}, \ldots, q_{k}$ because any two incomparable elements are connected by a blue edge. By a result of Gritzmann et al. [GMPP], [PA, Lemma 14.7], this contains a noncrossing cycle of length $k$, triangulated from a vertex.

\section{Paths-Proof of Theorem $\mathbf{1 . 2}$}

Let $G$ be a complete geometric graph whose edges are colored red and blue. Suppose that $G$ contains no monochromatic noncrossing path of length $m$. To establish Theorem 1.2, it is enough to prove that $G$ has at most $O\left(\mathrm{~m}^{3 / 2}\right)$ vertices.

Suppose without loss of generality that no two vertices lie on a vertical line. For each vertex $v$ of $G$, let $b(v)$ (resp. $r(v)$ ) denote the length of the longest $x$-monotone blue (resp. red) path in $G$ whose rightmost vertex is $v$.

Since every monotone path is noncrossing, $b(v)$ and $r(v)$ are integers between 0 and $m-1$. Observe that $(b(v), r(v)) \neq\left(b\left(v^{\prime}\right), r\left(v^{\prime}\right)\right)$ for $v \neq v^{\prime}$. Indeed, if $v \neq v^{\prime}$ are two vertices of $G$ such that $v^{\prime}$ lies to the right of $v$, then $b\left(v^{\prime}\right)>b(v)$ (if $v v^{\prime}$ is colored blue) 
or $r\left(v^{\prime}\right)>r(v)$ (if $v v^{\prime}$ is colored red). As a consequence, $G$ has at most $m^{2}$ vertices. The following more careful analysis gives a better bound.

Define $\mathcal{U}$ as the set of pairs $(b, r)$ with $(b, r)=(b(u), r(u))$ for some vertex $u$ of $G$. For $0 \leq b_{1} \leq b_{2} \leq m$ and $0 \leq r_{1} \leq r_{2} \leq m$, define a subset $\operatorname{Rect}\left(b_{1}, b_{2}, r_{1}, r_{2}\right)$ of $\mathcal{U}$ by

$$
\operatorname{Rect}\left(b_{1}, b_{2}, r_{1}, r_{2}\right)=\left\{(b, r) \in \mathcal{U} \mid b_{1} \leq b<b_{2}, r_{1} \leq r<r_{2}\right\} .
$$

Every pair $(b, r), 0 \leq b \leq m, 0 \leq r \leq m$, defines a partition of $\mathcal{U}$ into the following four subsets:

$$
\begin{array}{ll}
Q^{++}(b, r)=\operatorname{Rect}(b, m, r, m), & Q^{+-}(b, r)=\operatorname{Rect}(b, m, 0, r), \\
Q^{-+}(b, r)=\operatorname{Rect}(0, b, r, m), & Q^{--}(b, r)=\operatorname{Rect}(0, b, 0, r) .
\end{array}
$$

The key observation is the following.

Lemma 3.1. For any $t \in\{1,2, \ldots, 2 m-1\}$, there are two integers $b(t), r(t) \in$ $\{0,1, \ldots, m\}$ such that $b(t)+r(t)=t$ and

$$
\left|Q^{+-}(b(t), r(t)) \cup Q^{-+}(b(t), r(t))\right| \leq 3 m .
$$

Before giving the proof of Lemma 3.1, we finish the proof of Theorem 1.2. Obviously, it suffices to show that $|\mathcal{U}|=O\left(\mathrm{~m}^{3 / 2}\right)$.

Suppose for simplicity that $s=\sqrt{2 m}$ is an integer. Somewhat inaccurately, we shorten the notation $Q^{++}(b(t), r(t))$ to $Q^{++}(t)$. We also shorten $Q^{+-}(b(t), r(t)), Q^{-+}(b(t), r(t))$, and $Q^{--}(b(t), r(t))$ analogously. We have

$$
\mathcal{U}=\bigcup_{t=s, 2 s, \ldots, s^{2}-s}\left(Q^{+-}(t) \cup Q^{-+}(t)\right) \cup \bigcup_{t=0, s, 2 s, \ldots, s^{2}-s}\left(Q^{++}(t) \cap Q^{--}(t+s)\right),
$$

because each pair $(b, r) \in \mathcal{U}$ with $k s \leq b+r<(k+1) s$ lies in the first union (in a term with $t=k s$ or with $t=(k+1) s)$ or in the term $Q^{++}(k s) \cap Q^{--}(k s+s)$ of the second union. Consequently,

$$
|\mathcal{U}| \leq \sum_{t=s, 2 s, \ldots, s^{2}-s}\left|Q^{+-}(t) \cup Q^{-+}(t)\right|+\sum_{t=0, s, 2 s, \ldots, s^{2}-s}\left|Q^{++}(t) \cap Q^{--}(t+s)\right| .
$$

By Lemma 3.1, each term in the first sum is at most $3 m$, and it is easy to check that each term in the second sum is at most $(s / 2)^{2}=m / 2$. It follows that

$$
|\mathcal{U}| \leq(s-1) \cdot 3 m+s \frac{m}{2}=O\left(m^{3 / 2}\right) .
$$

It remains to prove Lemma 3.1 .

Proof of Lemma 3.1. Suppose, e.g., that $t \leq m$ (the case $t>m$ can be treated similarly). For each $b=0,1, \ldots, t$, denote

$$
\Delta(b)=\left|Q^{-+}(b, t-b)\right|-\left|Q^{+-}(b, t-b)\right| .
$$


It follows from

$$
\Delta(0) \leq 0 \leq \Delta(m)
$$

and from

$|\Delta(b+1)-\Delta(b)|=|\operatorname{Rect}(b, b+1,0, m)|+|\operatorname{Rect}(0, m, t-b-1, t-b)| \leq m+m=2 m$

that there is a $b_{0} \in\{0, \ldots, m\}$ with

$$
\left|\Delta\left(b_{0}\right)\right| \leq m .
$$

We now show that the lemma holds with $b(t)=b_{0}$ and $r(t)=t-b_{0}$. Suppose this is not true, i.e.,

$$
\left|Q^{+-}(t)\right|+\left|Q^{-+}(t)\right| \geq 3 m+1
$$

Then $|\Delta(b(t))| \leq m$ gives

$$
\left|Q^{+-}(t)\right| \geq m+1, \quad\left|Q^{-+}(t)\right| \geq m+1 .
$$

Consequently, there is a vertical line $\ell$ such that a set $V_{1}$ of at least $(m+1) / 2$ vertices to the left of $\ell$ corresponds to a subset of $Q^{+-}(t)$ or $Q^{-+}(t)$, and a set $V_{2}$ of at least $(m+1) / 2$ vertices to the right of $\ell$ corresponds to a subset of $Q^{-+}(t)$ or $Q^{+-}(t)$, respectively. All edges of the complete bipartite geometric graph $\left(V_{1} \cup V_{2}, V_{1} \times V_{2}\right)$ are colored by the same color: red, if $V_{1}$ corresponds to elements of $Q^{+-}(t)$, and blue, otherwise. It is well known (e.g., see $\left.\left[\mathrm{AGH}^{+}\right]\right)$that if $V_{1},\left|V_{1}\right| \geq(m+1) / 2$, is separated by a line from $V_{2}$, $\left|V_{2}\right| \geq(m+1) / 2$, then the graph $\left(V_{1} \cup V_{2}, V_{1} \times V_{2}\right)$ contains a noncrossing path of length $m-$ a contradiction.

In Theorem 1.2 the edges of a complete geometric graph $G$ are colored by two colors. If they are colored by $r \geq 2$ colors, we obtain the following.

Theorem 3.2. Every complete geometric graph of $n$ vertices whose edges are colored by $r \geq 2$ colors, contains a noncrossing path of length $\Omega\left(n^{2 /(2 r-1)}\right)$, all of whose edges are of the same color.

Proof. Let the edges of $G$ be colored by $1,2, \ldots, r$. Suppose that $G$ contains no noncrossing path of length $m$. For each color $i$, define a function $f_{i}$ on the vertex set of $G$ such that $f_{i}(v)$ is the length of the longest $x$-monotone monochromatic path of color $i$, whose rightmost point is $v$. Thus, for any vertex $v$, we have an $r$-tuple $f(v)=\left(f_{1}(v), \ldots, f_{r}(v)\right)$ of integers between 0 and $m-1$. Of course, $f(v) \neq f\left(v^{\prime}\right)$ for $v \neq v^{\prime}$, since $f(v)$ and $f\left(v^{\prime}\right)$ differ in the $i$ th coordinate, where $i$ is the color of the edge $v v^{\prime}$. We now show by induction on $r$ that the number of vertices of $G$ is at most $O\left(m^{r-1 / 2}\right)$. For $r=2$, this was shown in the proof of Theorem 1.2. Suppose now that $r>2$ and that the statement holds for $r-1$. Define $\mathcal{U}$ as the set of all $r$-tuples $f(v)$, where $v$ is a vertex of $G$. The $\operatorname{set} \mathcal{U}$ is a disjoint union of $m$ sets $\mathcal{U}_{j}, j=0,1, \ldots, m-1$, where $\mathcal{U}_{j}$ consists of the $r$-tuples of $\mathcal{U}$ whose last coordinate is $j$. For each $j$, all edges between the vertices corresponding to elements of $\mathcal{U}_{j}$ are colored by colors $1,2, \ldots, r-1$. By the inductive assumption, the size of $\mathcal{U}_{j}$ is at most $O\left(m^{r-3 / 2}\right)$. Consequently, the size of $\mathcal{U}$ is at most $O\left(m^{r-1 / 2}\right)$, and the result follows. 


\section{Paths in Convex Geometric Graphs-Proof of Theorems 1.3 and 1.4}

The proof of Theorem 1.3 is based on an unpublished result of Perles (Theorem 1.4), whose proof is as follows.

Proof of Theorem 1.4. Let $G$ be a convex geometric graph with vertices $u_{1}, u_{2}, \ldots, u_{n}$ in clockwise order and with $|E(G)|=m>\lfloor(k-1) n / 2\rfloor$. For convenience, let $u_{0}=u_{n}$ and $u_{n+i}=u_{i}$.

Fix $E_{0}=\emptyset$, and define $E_{1}, E_{2}, \ldots, E_{m}$ inductively, as follows. Pick an edge $e_{i} \in$ $\bar{E}_{i-1}=E(G) \backslash E_{i-1}$ such that one of the closed half-planes determined by $e_{i}$ does not fully contain any element of $E(G) \backslash\left(E_{i-1} \cup\left\{e_{i}\right\}\right)$. (Such an edge is called an extreme edge with respect to $\bar{E}_{i-1}$.) Let $E_{i}=E_{i-1} \cup\left\{e_{i}\right\}$.

We also define some nonnegative integers $c_{i}\left(u_{j}\right)$ and $d_{i}\left(u_{j}\right)$. For any vertex $u_{j}$, let $I=$ $I\left(u_{j}\right)$ be the smallest integer such that $E_{I}$ contains all edges of $G$ incident to $u_{j}$. Suppose first that $i<I$. Then, starting at $u_{j}$ and visiting the vertices of $G$ in clockwise order, let $u_{p}$ and $u_{q}$ be the first and the last vertex such that $u_{j} u_{p}, u_{j} u_{q} \in \bar{E}_{i}$. Let $c_{i}\left(u_{j}\right)\left(\operatorname{resp} . d_{i}\left(u_{j}\right)\right)$ denote the length of the longest noncrossing path in $E_{i}$ starting at $u_{j}$, all of whose vertices are in $\left\{u_{j}, u_{j+1}, \ldots, u_{p-1}\right\}$ (resp. $\left\{u_{q+1}, u_{q+2}, \ldots, u_{j}\right\}$ ). Next, if $i=I$, then $e_{i}=u_{j} u_{p}$ for some vertex $u_{p}$. Since $e_{i}$ is an extremal edge with respect to $\bar{E}_{i-1}$, we may suppose, by symmetry, that $\bar{E}_{i}$ has no element on the vertex set $\left\{u_{j}, u_{j+1}, \ldots, u_{p-1}, u_{p}\right\}$. If this is the case, then let $c_{i}\left(u_{j}\right)$ (resp. $\left.d_{i}\left(u_{j}\right)\right)$ denote the length of the longest noncrossing path in $E_{i}$ starting at $u_{j}$, all of whose vertices lie in $\left\{u_{j}, u_{j+1}, \ldots, u_{p}\right\}$ (resp. $\left\{u_{p+1}, u_{p+2}, \ldots, u_{j}\right\}$ ). (Otherwise we change the orientation.) Finally, if $i>I$, then put $c_{i}\left(u_{j}\right)=c_{I}\left(u_{j}\right)$ and $d_{i}\left(u_{j}\right)=d_{I}\left(u_{j}\right)$

Claim 4.1. For every $0 \leq i \leq m$,

$$
\sum_{j=1}^{n} c_{i}\left(u_{j}\right)+\sum_{j=1}^{n} d_{i}\left(u_{j}\right) \geq 2\left|E_{i}\right| .
$$

The claim is clearly true for $i=0$. Let $0<i \leq m$, and assume that the assertion has already been proved for $i-1$. Suppose that the endpoints of $e_{i}$ are $u_{j}$ and $u_{l}$. Since $c_{i}(u) \geq c_{i-1}(u)$ and $d_{i}(u) \geq d_{i-1}(u)$ for every fixed vertex $u$, it is enough to prove that

$$
c_{i}\left(u_{j}\right)+d_{i}\left(u_{j}\right)+c_{i}\left(u_{l}\right)+d_{i}\left(u_{l}\right) \geq c_{i-1}\left(u_{j}\right)+d_{i-1}\left(u_{j}\right)+c_{i-1}\left(u_{l}\right)+d_{i-1}\left(u_{l}\right)+2 .
$$

However, we either have $c_{i}\left(u_{j}\right)>d_{i-1}\left(u_{l}\right)$ and $d_{i}\left(u_{l}\right)>c_{i-1}\left(u_{j}\right)$, or we have $c_{i}\left(u_{l}\right)>$ $d_{i-1}\left(u_{j}\right)$ and $d_{i}\left(u_{j}\right)>c_{i-1}\left(u_{l}\right)$, depending on which side of $e_{i}=u_{j} u_{l}$ contains no edge of $\bar{E}_{i}$, and the statement follows.

Now it is easy to complete the proof of the first part of Theorem 1.4. Since $\left|E_{m}\right|>$ $\lfloor(k-1) n / 2\rfloor$, there is $1 \leq j \leq n$ for which $c_{m}\left(u_{j}\right)+d_{m}\left(u_{j}\right) \geq k$. By the definition of $c_{m}$ and $d_{m}$, this means that there is a noncrossing path of length $k$, passing through $u_{j}$.

The following construction shows that the bound in Theorem 1.4 is tight. 


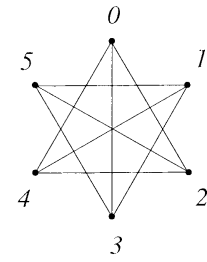

$n=6, k=4$

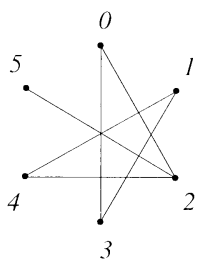

$n=6, k=3$

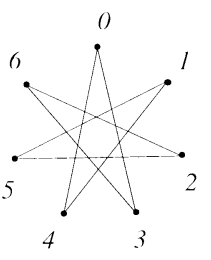

$n=7 . k=3$

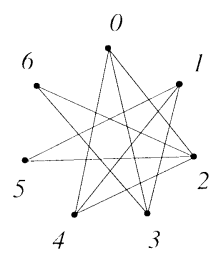

$n=7, l=1$

Fig. 1

Construction. Let $0,1, \ldots, n-1(\bmod n)$ denote the vertices of a regular $n$-gon.

(i) If $n \equiv k(\bmod 2)$, then connect each vertex to the $k-1$ vertices furthest from it.

(ii) If $n \neq k(\bmod 2)$, then connect each vertex $i$ to the $k-2$ vertices furthest from $i$, and, for $0 \leq i \leq\lfloor n / 2\rfloor-1$, also to $i+(n-k+1) / 2$.

In case (i), every edge has at least $(n-k) / 2$ vertices on both sides. In particular, this holds for the first and last edges of any noncrossing path. This implies that any such path misses at least $n-k$ vertices, so it is of length at most $k-1$.

In case (ii), suppose that $k+3 \leq n$. Consider any noncrossing path $P$. Call an edge of $P$ extremal if the whole path $P$ lies in one of the closed half-spaces determined by $P$. If $P$ has at least three extremal edges, then it has at most $n-3(n-k-1) / 2 \leq k$ vertices, showing that its length is at most $k-1$. However, if $P$ has only two extremal edges, then $P$ is a "zigzag," in particular, for each $j,\lfloor n / 2\rfloor \geq j \geq(n-k+1) / 2$, it has at most two edges of the form $(i, i+j)$. If at most one edge of $P$ is of the form $(i, i+(n-k+1) / 2)$, then $P$ contains at most $k-1$ edges. Otherwise, the first and the last edges of $P$ are of the form $(i, i+(n-k+1) / 2)$ and $(j, j+(n-k+1) / 2)$ with $0 \leq i<i+(n-k+1) / 2<j \leq\lfloor n / 2\rfloor-1$. In this case, the length of $P$ is at most

$$
2\left(j-\left(i+\frac{n-k+1}{2}\right)+1\right) \leq 2\left(\left\lfloor\frac{n}{2}\right\rfloor-\frac{n-k+1}{2}\right) \leq k-1,
$$

as required.

The case $n=k+1$ can be treated similarly.

We prove the following equivalent form of Theorem 1.3.

Theorem 4.2. Let $\mathcal{P}_{k}$ be the class of all noncrossing paths of length $k$. Then $R_{\mathrm{c}}\left(\mathcal{P}_{k}\right)=$ $2 k-1$ unless $k=1$.

Proof. First, we show that $R_{\mathrm{c}}\left(\mathcal{P}_{k}\right)>2 k-2$. Let $G$ be a convex geometric graph on $2 k-2$ vertices, and let $p, v_{1}, v_{2}, \ldots, v_{k-2}, q, u_{1}, u_{2}, \ldots, u_{k-2}$ be its vertices listed in clockwise order. For all $i, j$, color all edges $\left(v_{i}, v_{j}\right),\left(p, v_{i}\right)$, and $\left(q, v_{i}\right)$ blue; $\left(u_{i}, u_{j}\right)$, $\left(p, u_{i}\right)$, and $\left(q, u_{i}\right)$ red; $\left(v_{i}, u_{j}\right)$ red if $i+j$ is odd and blue if $i+j$ is even. The edge $(p, q)$ can have any color. It is not difficult to check that this graph contains no noncrossing monochromatic path of length $k$. 
For the upper bound, first let $k$ be even and take a convex complete geometric graph on $2 k-1$ vertices, all of whose edges are colored red or blue. This graph has $(2 k-1)(k-1)$ edges, so one of the color classes (say, red) contains at least $\lceil(2 k-1)(k-1) / 2\rceil>$ $\lfloor(2 k-1)(k-1) / 2\rfloor$ edges. Therefore, by Theorem 1.4 , there is a noncrossing red path of length $k$.

If $k$ is odd, then we have to be more careful. Suppose there is a two-coloring of the edges of a complete geometric graph $G$ on $2 k-1$ vertices without a noncrossing path of length $k$. It follows from Theorem 1.4 that $(2 k-1)(k-1) / 2$ edges are red and the same number of edges are blue. Let $G_{\mathrm{b}}$ denote the subgraph of $G$ obtained from $G$ by removing all red edges. Analyzing the proof of Theorem 1.4 for $G_{\mathrm{b}}$, we obtain that (in the notation of the proof of Theorem 1.4)

$$
\sum_{j=1}^{n} c_{i}\left(u_{j}\right)+\sum_{j=1}^{n} d_{i}\left(u_{j}\right)=2\left|E_{i}\right|, \quad i=1,2, \ldots, m .
$$

Consequently,

$$
c_{i}\left(u_{j}\right)+d_{i}\left(u_{j}\right)+c_{i}\left(u_{l}\right)+d_{i}\left(u_{l}\right)=c_{i-1}\left(u_{j}\right)+d_{i-1}\left(u_{j}\right)+c_{i-1}\left(u_{l}\right)+d_{i-1}\left(u_{l}\right)+2 .
$$

(Otherwise, we would have $c_{m}\left(u_{j}\right)+d_{m}\left(u_{j}\right) \geq k$ for some $j$, and we could find a noncrossing path of length $k$.) This means, for example, that if $e_{i}=u_{j} u_{l}$ and $\bar{E}_{i}$ has no element on the vertex set $\left\{u_{j}, u_{j+1}, \ldots, u_{l-1}, u_{l}\right\}$, then $c_{i}\left(u_{j}\right)=d_{i-1}\left(u_{l}\right)+1, d_{i}\left(u_{l}\right)=$ $c_{i-1}\left(u_{j}\right)+1$.

Looking at all possible ways of how we may arrange the edges of $G_{\mathrm{b}}$ in the sequence $e_{1}, e_{2}, \ldots, e_{m}$, we obtain the following proposition.

Proposition 4.3. Let $v, w \in\{1,2, \ldots, 2 k-1\}$.

(a) If $u_{v} u_{w}$ is blue, then at least one of the longest blue noncrossing paths starting at $u_{v}$, all of whose vertices lie in $\left\{u_{v}, u_{v+1}, \ldots, u_{w}\right\}$, contains the edge $u_{v} u_{w}$.

(b) If $u_{v} u_{w}$ is blue, then at least one of the longest blue noncrossing paths starting at $u_{w}$, all of whose vertices lie in $\left\{u_{v}, u_{v+1}, \ldots, u_{w}\right\}$, contains the edge $u_{v} u_{w}$.

(c) If $u_{v} u_{w}$ is red, then at least one of the longest blue noncrossing paths starting at $u_{v}$, all of whose vertices lie in $\left\{u_{v}, u_{v+1}, \ldots, u_{w}\right\}$, does not contain $u_{w}$.

(d) If $u_{v} u_{w}$ is red, then at least one of the longest blue noncrossing paths starting at $u_{w}$, all of whose vertices lie in $\left\{u_{v}, u_{v+1}, \ldots, u_{w}\right\}$, does not contain $u_{v}$.

Exchanging the roles of the colors, a similar statement can be formulated about red paths in the graph $G_{\mathrm{r}}$ obtained from $G$ by deleting all blue edges.

Consider the $2 k-1$ edges $u_{i} u_{i+1}$. At least $k$ of them are colored by the same color (blue, say). Let $u_{p} u_{p+1} \cdots u_{q}$ be the longest noncrossing blue path using some of the edges $u_{i} u_{i+1}$ (certainly, its length is at least 2). First, observe that Proposition 4.3(c) yields that all edges within $\left\{u_{p}, u_{p+1}, \ldots, u_{q}\right\}$ are blue. Indeed, if $u_{s} u_{t}, p \leq s<t \leq q$, was a red edge such that all other edges $u_{s^{\prime}} u_{t^{\prime}}, s \leq s^{\prime}<t^{\prime} \leq t$, were blue, then Proposition 4.3(c) would be false with $u=s$ and $w=t$. This implies that the blue path $u_{p} u_{p+1} \cdots u_{q}$ cannot visit all or all but one of the vertices of $G$. By the maximality of this path, the edges $u_{p-1} u_{p}$ and $u_{q} u_{q+1}$ are red. It follows from the the dual of Proposition 4.3(a) and (c) with $v=p-1$ and $w=q+1$ that $u_{p} u_{q+1}$ is blue. Analogously, $u_{p-1} u_{q}$ is also blue. 
Suppose now that some of the edges $u_{p-1} u_{r}, r=p+1, p+2, \ldots, q-1$, are red. Take one of them for which $r$ is maximum. Then Proposition 4.3(b) would be violated for $v=p-1$ and $w=r+1$. Thus, all edges $u_{p-1} u_{r}, r=p+1, p+2, \ldots, q$, are blue. Analogously, all edges $u_{q+1} u_{r}, r=p, p+1, \ldots, q-1$, are also blue.

The edge $u_{p-2} u_{p-1}$ cannot be red. Otherwise, starting with the longest noncrossing red path $u_{p} u_{p-1} \cdots$ and using a similar argument as above, we would conclude that $u_{p-1} u_{p+1}$ must be red, but we already know that it is blue. Thus, $u_{p-2} u_{p-1}$ is blue, and we get a contradiction with Proposition 4.3(a) or (c) for $v=p-2$ and $w=p+1$.

\section{General Estimates-Proof of Theorem 1.5}

For any set of $n$ points $P$ in the plane, an $i$-element subset of $P$ is called an $i$-set if it can be obtained by intersecting $P$ with an open half-plane. It is easy to see that all $i$-sets can be generated by the following procedure [ELSS]: Take an oriented line $\ell$ passing through precisely one point $p \in P$ and having $i$ elements of $P$ on its left side. Rotate $\ell$ around $p$ in the clockwise direction until it hits another point $q \in P$, and then continue the rotation around $q$, etc. Whenever $\ell$ passes through only one element of $P$, the points lying on its left side form an $i$-set.

Any geometric subgraph $K_{R(\mathcal{G})}$ induced by an $R(\mathcal{G})$-element subset of $P$ contains a subgraph of the class $\mathcal{G}$, all of whose edges are of the same color. If these edges are red (blue), we say that the type of the corresponding subset is red (blue). Note that a set may have both types.

Lemma 5.1. Let $\mathcal{G}$ be any class of geometric graphs. Then $R(2 \mathcal{G}) \leq 2 R(\mathcal{G})+1$.

Proof. Let $N=2 R(\mathcal{G})+1$ and let $P$ be the vertex set of a complete geometric graph $K_{N}$ whose edges are colored by red and blue.

If all $R(\mathcal{G})$-sets of $P$ have the same type, then take two disjoint $R(\mathcal{G})$-sets. Both contain a monochromatic geometric subgraph belonging to $\mathcal{G}$, so their union is a monochromatic member of $2 \mathcal{G}$.

On the other hand, if there are two $R(\mathcal{G})$-sets of different types, then we can also find two $R(\mathcal{G})$-sets, $A$ and $B$, of different types such that $|A \cup B|=R(\mathcal{G})+1$ and that $P \backslash(A \cup B)$ is also an $R(\mathcal{G})$-set. The type of $P \backslash(A \cup B)$ will coincide with the type of $A$ or $B$.

Proof of Theorem 1.5. Part (i) is an immediate corollary of Lemma 5.1.

Let $\mathcal{G}$ be a (fixed) class of geometric graphs. Let $r=r(k, l)$ be the smallest number with the property that every complete geometric graph with $r$ vertices, whose edges are colored by red and blue, contains either a subgraph belonging to $k \mathcal{G}$, all of whose edges are red, or a subgraph belonging to $l \mathcal{G}$, all of whose edges are blue. So, $R=R(\mathcal{G})=r(1,1)$, $R(k \mathcal{G})=r(k, k)$. 
Lemma 5.2. For $k \geq l \geq 1$,

$$
r(k, l)=r(l, k) \leq(R+1) k+\left\lceil\frac{R+1}{2}\right\rceil l-\left\lceil\frac{R+1}{2}\right\rceil .
$$

Proof. By symmetry, clearly $r(k, l)=r(l, k)$. We proceed by induction on $k$ and $l$. First we show that $r(k, 1) \leq k R$. Let $G$ be a complete geometric graph with $k R$ vertices, whose edges are colored by red and blue. Suppose that no two vertices determine a vertical line. Then we can choose $k-1$ vertical lines that divide the vertex set of $G$ into $k$ equal parts. Since each part contains $R$ of the vertices, the subgraph spanned by every part contains a monochromatic copy of a geometric graph from the class $\mathcal{G}$. Either all of them are red or one of them is blue. Therefore, the lemma holds for any pair $(k, l)$, where either $k=1$ or $l=1$.

Let $k \geq l$ be fixed, and suppose that we already know that the lemma is true for every pair $\left(k^{\prime}, l^{\prime}\right) \neq(k, l)$, where $k^{\prime} \leq k, l^{\prime} \leq l$.

Consider a complete geometric graph $G$ with vertex set $V$, whose edges are colored red and blue, and

$$
|V|=(R+1) k+\left\lceil\frac{R+1}{2}\right\rceil l-\left\lceil\frac{R+1}{2}\right\rceil .
$$

By Theorem 1.5(i), for any $n \geq 1$, if $f(n)=2^{n}(R+1)-1 \leq|V|$, then any geometric subgraph induced by an $f(n)$-set contains a monochromatic subgraph from the class $2^{n} \mathcal{G}$. Its color will be the type of the corresponding $f(n)$-set.

If there are two $f(n)$-sets of different types, we can cut off an $(f(n)+1)$-set which contains an $f(n)$-set of both types. Since

$$
(R+1) k+\left\lceil\frac{R+1}{2}\right\rceil l-2^{n}(R+1) \geq(R+1)\left(k-2^{n}\right)+\left\lceil\frac{R+1}{2}\right\rceil\left(l-2^{n}\right),
$$

we can apply the induction hypothesis for the subgraph induced by the rest of the vertices, with $k^{\prime}=k-2^{n}, l^{\prime}=l-2^{n}$.

So we can assume that, for all $n$, all $f(n)$-sets are of the same type, provided $f(n)=$ $2^{n}(R+1)-1 \leq|V|$. Suppose that, for $n_{1}<n_{2}$, the $f\left(n_{1}\right)$ - and $f\left(n_{2}\right)$-sets are of different types. Then there exists an $n_{1} \leq n<n_{2}$ such that the $f(n)$ - and $f(n+1)$-sets are also of different types. Cut off an $f(n+1)$-set. Since it contains an $f(n)$-set, the subgraph induced by the $f(n+1)$-set contains a blue (resp. red) subgraph from the class $2^{n+1} \mathcal{G}$ and a red (resp. blue) subgraph from the class $2^{n} \mathcal{G}$.

If the $f(n+1)$-sets are of type blue, then either $l \leq 2^{n+1}$ and we are done, or we can apply the induction hypothesis for the graph induced by the remaining vertices with $k^{\prime}=k-2^{n}$ and $l^{\prime}=l-2^{n+1}$ since it is easy to check that

$$
(R+1) k+\left\lceil\frac{R+1}{2}\right\rceil l-2^{n+1}(R+1) \geq(R+1)\left(k-2^{n}\right)+\left\lceil\frac{R+1}{2}\right\rceil\left(l-2^{n+1}\right) .
$$

On the other hand, if the $f(n+1)$-sets are of type red, then we can assume that $k>2^{n+1}$ and $l>2^{n}$ and we can apply the induction hypothesis with $k^{\prime}=k-2^{n+1}$ and 
$l^{\prime}=l-2^{n}$ since

$$
(R+1) k+\left\lceil\frac{R+1}{2}\right\rceil l-2^{n+1}(R+1) \geq(R+1)\left(k-2^{n+1}\right)+\left\lceil\frac{R+1}{2}\right\rceil\left(l-2^{n}\right)
$$

and

$$
(R+1) k+\left\lceil\frac{R+1}{2}\right\rceil l-2^{n+1}(R+1) \geq(R+1)\left(l-2^{n}\right)+\left\lceil\frac{R+1}{2}\right\rceil\left(k-2^{n+1}\right) .
$$

Therefore, in what follows we assume that, for all $n$, all $f(n)$-sets are of the same type. For simplicity we assume that they are of type red. The case where these sets are of type blue can be settled very similarly. Write $k$ in base 2 , that is, $k=2^{n_{1}}+2^{n_{2}}+\cdots+2^{n_{a}}$, $n_{1}<n_{2}<\cdots<n_{a}$. Let $V_{0}=V$. By the assumption, every $f\left(n_{1}\right)$ - and $f\left(n_{2}\right)$-set of $V_{0}$ are of type red. Let $S_{1}$ be an $f\left(n_{1}\right)$-set and let $V_{1}=V_{0} \backslash S_{1}$. If every $f\left(n_{2}\right)$ - and $f\left(n_{3}\right)$-set of $V_{1}$ is of type red, then let $S_{2}$ be such an $f\left(n_{2}\right)$-set and let $V_{2}=V_{1} \backslash S_{2}$. In general, if $V_{i}$ has already been defined by this procedure and every $f\left(n_{i+1}\right)$ - and $f\left(n_{i+2}\right)$-set of $V_{i}$ is of type red, then let $S_{i+1}$ be any $f\left(n_{i+1}\right)$-set and let $V_{i+1}=V_{i} \backslash S_{i+1}$. Otherwise, if not all $f\left(n_{i+1}\right)$-sets or not all $f\left(n_{i+2}\right)$-sets of $V_{i}$ are of type red, or $i=a-1$, then stop and let $j=i$.

If $j=a-1$, then $V_{a-1}$ still has an $f\left(n_{a}\right)$-set, $S_{a}$ of type red, and $S_{1} \cup S_{2} \cup \cdots \cup S_{a}$ contains a red subgraph from the class $k \mathcal{G}$ and we are done. Suppose $j<a-1$. We distinguish two cases.

Case a: There is an $f\left(n_{j+1}\right)$-set of $V_{j}$ of type blue. Since all $f\left(n_{j+1}\right)$-sets of $V_{j-1}$ were of type red, at least one of the $f\left(n_{j+1}\right)$-sets of $V_{j}$ is of type red. Therefore, we can find an $\left(f\left(n_{j+1}\right)+1\right)$-set, $S_{j+1}$, which contains an $f\left(n_{j+1}\right)$-set of both types. $\mid S_{1} \cup S_{2} \cup \cdots \cup$ $S_{j+1} \mid \leq\left(2^{n_{1}}+2^{n_{2}}+\cdots+2^{n_{j+1}}\right)(R+1)$ and $S_{1} \cup S_{2} \cup \cdots \cup S_{j+1}$ contains a red subgraph from the class $\left(2^{n_{1}}+2^{n_{2}}+\cdots+2^{n_{j+1}}\right) \mathcal{G}$ and a blue subgraph from the class $2^{n_{j+1}} \mathcal{G}$. If either $k \leq 2^{n_{1}}+2^{n_{2}}+\cdots+2^{n_{j+1}}$ or $l \leq 2^{n_{j+1}}$, then we are done. Otherwise, easy calculation shows that we can apply the induction hypothesis for the graph induced by the remaining vertices with $k^{\prime}=k-\left(2^{n_{1}}+2^{n_{2}}+\cdots+2^{n_{j+1}}\right)$ and $l^{\prime}=l-2^{n_{j+1}}$.

Case b: Every $f\left(n_{j+1}\right)$-set of $V_{i}$ is of type red, but there is an $f\left(n_{j+2}\right)$-set of $V_{j}$ of type blue. In this case, there exists an $n_{j+1} \leq n<n_{j+2}$ such that every $f(n)$-set of $V_{i}$ is of type red, but there is an $f(n+1)$-set of $V_{j}$ of type blue. Then let $S_{j+1}$ be an $f(n+1)$-set of $V_{j}$ of type blue. Clearly, it contains an $f(n)$-set of $V_{j}$, which is of type red. $\left|S_{1} \cup S_{2} \cup \cdots \cup S_{j+1}\right| \leq\left(2^{n_{1}}+2^{n_{2}}+\cdots+2^{n_{j}}+2^{n+1}\right)(R+1)$ and $S_{1} \cup S_{2} \cup \cdots \cup S_{j+1}$ contains a red subgraph from the class $\left(2^{n_{1}}+2^{n_{2}}+\cdots+2^{n_{j}}+2^{n}\right) \mathcal{G}$ and a blue subgraph from the class $2^{n+1} \mathcal{G}$. Again, easy calculation shows that we can proceed as in case a.

Return to the proof of Theorem 1.5. Part (ii) is an immediate corollary of Lemma 5.2. In part (iii), if all $R_{\mathrm{c}}(\mathcal{G})$-sets have the same type then, using the convexity of the geometric graph, we can take $k$ disjoint $R_{\mathrm{c}}(\mathcal{G})$-sets and we get a monochromatic geometric graph from $k \mathcal{G}$.

If there are two $R(\mathcal{G})$-sets of different types, we can proceed by induction as in part (ii). 


\section{Triangles-Proof of Theorem 1.6}

The proof is quite technical, therefore we sketch here only the main ideas.

It is sufficient to show that $R_{\Delta}(k)>7 k-7$ for every $k$. Indeed, assume that this is true, but $R_{\Delta}\left(k_{0}\right) \leq 7 k_{0}-2$ for some integer $k_{0}$. Then, applying Theorem 1.5(i) with $\mathcal{G}=k_{0} \mathcal{T}$, we would obtain

$$
\begin{aligned}
R_{\Delta}\left(8 k_{0}\right) & =R\left(8 k_{0} \mathcal{T}\right) \leq\left(R\left(k_{0} \mathcal{T}\right)+1\right) 8-1 \\
& =\left(R_{\Delta}\left(k_{0}\right)+1\right) 8-1 \leq\left(7 k_{0}-1\right) 8-1=7\left(8 k_{0}\right)-9,
\end{aligned}
$$

a contradiction.

Construction. Let $k$ be a positive integer, $n=7 k-7$. Let $0,1, \ldots, 7 k-8$ denote the vertices of a regular $n$-gon, in clockwise order. If two vertices are separated by $m-1$ others, then we say that the length of the edge connecting them is $m(m \leq\lfloor n / 2\rfloor)$. A vertex is colored red if $i \equiv 0,1,3$, or $5(\bmod 7)$, and blue otherwise.

We color the edges $i j(0 \leq i \neq j \leq n-1)$ according to the following rules. Let $\lambda$ denote the length of $i j$.

(a) If $\lambda \equiv 1,4$, or $6(\bmod 7)$, let $i j$ be blue.

(b) If $\lambda \equiv 2$ or $3(\bmod 7)$, let $i j$ be red.

(c) If $\lambda \equiv 5(\bmod 7)$, let $i j$ be colored with the color of its starting point in the clockwise direction.

(d) If $\lambda \equiv 0(\bmod 7)$, let $i j$ be colored with the color other than the color of $i+$ $1(\bmod 7)$.

The case $k=3$ is depicted in Fig. 2. To see that this graph does not contain $k$ pairwise noncrossing blue triangles, we need the following lemma that can be proved by induction. By an $\operatorname{arc}$ we mean a set of consecutive vertices.

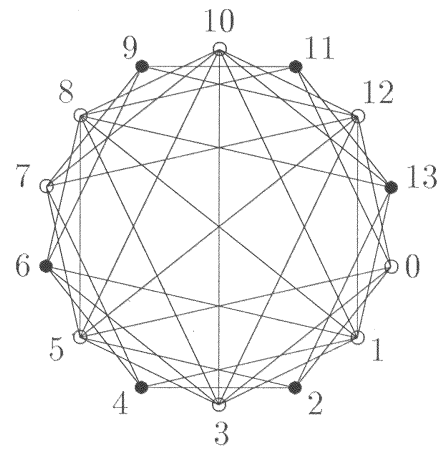

Red edges

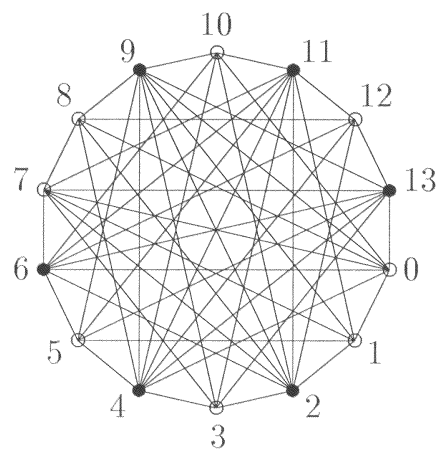

Blue edges

Fig. 2. 
Lemma 6.1. Suppose that an arc I of $m \leq n / 2$ vertices contains the vertices of $h$ pairwise noncrossing blue triangles. Then $m \geq 7 h-1$. Moreover, if $m=7 \mathrm{~h}-1$, then the starting point of I is blue.

Consider a maximal set of pairwise noncrossing blue triangles. Suppose first that the points can be partitioned into three arcs, $I_{1}, I_{2}, I_{3}$, with $m_{i}=\left|I_{i}\right| \leq n / 2$ such that the vertices of each of these triangles belong to the same arc. Let $k_{i}$ denote the number of triangles whose vertices belong to $I_{i}$. By Lemma 6.1, we have

$7 n-7=m_{1}+m_{2}+m_{3} \geq\left(7 k_{1}-1\right)+\left(7 k_{2}-1\right)+\left(7 k_{3}-1\right)>7\left(k_{1}+k_{2}+k_{3}\right)-7$, and we can conclude that $k_{1}+k_{2}+k_{3}<n$.

Otherwise, there is a blue triangle so that the convex hull of its vertex set contains the center of our regular $n$-gon. Its vertices, $p, q, r$, partition the remaining set of points into three arcs of lengths $m_{1}, m_{2}, m_{3}$ containing $k_{1}, k_{2}, k_{3}$ triangles, respectively. From Lemma 6.1 we obtain

$7 n-7=m_{1}+m_{2}+m_{3}+3 \geq\left(7 k_{1}-1\right)+\left(7 k_{2}-1\right)+\left(7 k_{3}-1\right)+3=7\left(k_{1}+k_{2}+k_{3}\right)$,

implying $n \geq k=k_{1}+k_{2}+k_{3}+1$. Suppose that $n=k$. Then we have equality in the previous inequalities, therefore the length of each side of triangle $p q r$ is divisible by 7 . On the other hand, by Lemma 6.1, all of the points $p+1, q+1, r+1$ are blue, so $p q r$ is a monochromatic red triangle, a contradiction.

The existence of $n$ noncrossing red triangles can be excluded by a similar argument.

\section{References}

[AGH ${ }^{+}$] M. Abellanas, J. García, G. Hernández, M. Noy, and P. Ramos, Bipartite embeddings of trees in the plane, in: Graph Drawing '96 (S. North, ed.), Lecture Notes in Computer Science, vol. 1190, Springer-Verlag, Berlin, 1997, pp. 1-10.

[B] S.A. Burr, Generalized Ramsey theory for graphs - a survey, in: Graphs and Combinatorics (R. Bari and F. Harary, eds.), Lecture Notes in Mathematics, vol. 406, Springer-Verlag, Berlin, 1974, pp. 5275.

[BES] S.A. Burr, P. Erdős, and J.H. Spencer, Ramsey theorems for multiple copies of graphs, Transactions of the American Mathematical Society 209 (1975), 87-99.

[D] R.P. Dilworth, A decomposition theorem for partially ordered sets, Annals of Mathematics 51 (1950), 161-166.

[ELSS] P. Erdős, L. Lovász, A. Simmons, and E.G. Straus, Dissection graphs of planar point sets, in: A Survey of Combinatorial Theory (G. Srivastava, ed.), North-Holland, Amsterdam, 1973, pp. 139-149.

[GMPP] P. Gritzmann, B. Mohar, J. Pach, and R. Pollack, Embedding a planar triangulation with vertices at specified points (solution to problem E3341), American Mathematical Monthly 98 (1991), 165-166.

[GRS] R.L. Graham, B.L. Rothschild, and J.H. Spencer, Ramsey Theory, 2nd edn., Wiley, New York, 1990.

[KPT] Gy. Károlyi, J. Pach, and G. Tóth, Ramsey-type results for geometric graphs, I, in: Proceedings of the 12th Annual ACM Symposium on Computational Geometry, 1996, pp. 359-365. Also in Discrete and Computational Geometry 18 (1997), 247-255.

[KPTT] Gy. Károlyi, J. Pach, G. Tardos, and G. Tóth, An algorithm for finding many disjoint monochromatic edges in a complete 2-colored geometric graph, in: Intuitive Geometry (I. Bárány and K. Böröczky, eds.), Bolyai Society Mathematical Studies, vol. 6, Budapest, 1997, pp. 367-372.

[PA] J. Pach and P.K. Agarwal, Combinatorial Geometry, Wiley, New York, 1995.

Received March 25, 1997, and in revised form March 5, 1998. 